\title{
In-vivo Measurements of Ischiofemoral Space in Recreationally Active Subjects during Dynamic Activities: A High-speed Dual Fluoroscopy Study
}

\author{
Penny R. Atkins, BS ${ }^{1,2}$, Niccolo M. Fiorentino, PhD ${ }^{1}$, Stephen K. Aoki, MD ${ }^{1}$, Christopher L. \\ Peters, MD ${ }^{1,2}$, Travis G. Maak, MD ${ }^{1}$, and Andrew E. Anderson, PhD $^{1,2,3,4}$ \\ ${ }^{1}$ Department of Orthopaedics, University of Utah, 590 Wakara Way Rm A100, Salt Lake City, UT \\ 84108, USA \\ ${ }^{2}$ Department of Bioengineering, University of Utah, 36 S. Wasatch Drive Rm 3100, Salt Lake City, \\ UT 84112, USA \\ ${ }^{3}$ Scientific Computing and Imaging Institute, University of Utah, $72 \mathrm{~S}$ Central Campus Drive Rm \\ 3750, Salt Lake City, UT 84112, USA \\ ${ }^{4}$ Department of Physical Therapy, University of Utah, 520 Wakara Way Suite 240, Salt Lake City, \\ UT 84108, USA
}

\section{Abstract}

Background-Ischiofemoral impingement (IFI) is a dynamic process, yet diagnosis is often based on static, supine images.

\begin{abstract}
Purpose-To couple 3D computed tomography (CT) models with dual fluoroscopy (DF) images to quantify in-vivo hip motion and ischiofemoral space (IFS) in asymptomatic subjects during weight-bearing activities and evaluate the relationship of dynamic measurements with sex, hip kinematics, and IFS measured from axial magnetic resonance imaging (MRI).

Study Design-Cross-sectional study; Level of evidence, 3.

Methods-Eleven young, asymptomatic adults (five females) were recruited. 3D reconstructions of the femur and pelvis were generated from MRI and CT images. Axial and 3D IFS were measured from supine MR images. In-vivo hip motion during weight-bearing activities was quantified using DF. Bone-to-bone distance between the lesser trochanter and ischium was measured dynamically. Minimum and maximum IFS were determined and evaluated against hip joint angles using a linear mixed effects model.
\end{abstract}

Results-Minimum IFS occurred during weighted external rotation for 10/11 subjects. Measurements of IFS from axial MRI (mean (95\% CI); 23.7 (19.9-27.9) $\mathrm{mm}$ ) were significantly greater than minimum IFS observed during external rotation $(10.8(8.4-13.7) \mathrm{mm}, \mathrm{p}<0.001)$, level walking (15.5 (11.4-19.7) mm, $\mathrm{p}=0.007)$, and incline walking (15.8 (11.6-20.2) $\mathrm{mm}, \mathrm{p}=0.004)$, but not for standing. IFS was reduced with extension $(\beta=0.64)$, adduction $(\beta=0.23)$ and external

Corresponding Author: Andrew E. Anderson, University of Utah Orthopaedics, 590 Wakara Way Rm A100, Salt Lake City, UT 84108, USA, Tel: +1 801587 5208, Andrew.Anderson@hsc.utah.edu. 
rotation angles $(\beta=0.22)(\mathrm{p}<0.001$ for all $)$ during the dynamic activities observed. IFS was smaller in females than males for standing (20.9 (19.4-22.3) vs. 30.4 (27.2-33.8) $\mathrm{mm}, \mathrm{p}=0.045)$, level walking (8.8 (7.5-9.9) vs. $21.1(18.7-23.5) \mathrm{mm}, \mathrm{p}=0.003)$, and incline walking activities (9.1 (7.4$10.8)$ vs. $21.3(18.8-24.1) \mathrm{mm}, \mathrm{p}=0.005)$. Joint angles between sexes were not significantly different for any of the dynamic positions of interest.

Conclusion-Minimum IFS during dynamic activities was smaller than axial MRI measurements. Compared to males, IFS in females was reduced during standing and walking, despite a lack of kinematic differences between sexes.

Clinical Relevance-The relationship between IFS and hip joint angles suggests that clinical exams and imaging should place the hip into greater extension, adduction, and external rotation, as IFS measured from static images, especially in a neutral orientation, may not accurately represent minimum IFS during dynamic motion. Nevertheless, this statement must be interpreted with caution, as only asymptomatic subjects were analyzed herein.

\section{Keywords}

hip; motion analysis; impingement; extra-articular; ischiofemoral

\section{INTRODUCTION}

Ischiofemoral impingement (IFI) is a recently recognized cause of extra-articular hip pain that is exacerbated by hip adduction, external rotation, and extension. Johnson originally identified IFI in patients after trauma or surgery, ${ }^{16}$ but more recently, IFI has been observed in patients without history of trauma, surgery or morphological deformities. ${ }^{27,} 28$ IFI diagnosis is often based on patient reported hip pain and evaluation of magnetic resonance (MR) images for quadratus femoris muscle edema. In an effort to establish quantitative guidelines for the diagnosis of IFI, several studies have measured ischiofemoral space (IFS) in both patient and asymptomatic populations using both MR imaging (MRI) and ultrasound imaging modalities and identified reduced IFS in the patient populations.

$2,6,9,12,24,26,29,32,33$

Johnson suggested that an IFS of $2 \mathrm{~cm}$ under slight adduction, external rotation, and extension should be considered normal; however, the measurement technique used was not described. ${ }^{16}$ More recently, MRI has been used to measure IFS. As described by Torriani et al., IFS is to be measured axially as the minimum distance between the medial cortex of the lesser trochanter and lateral cortex of the ischial tuberosity. ${ }^{32}$ Using this technique in either a neutral or internally rotated hip position, the mean IFS in asymptomatic controls has been reported between 17.6 and $30.6 \mathrm{~mm}$. , 6, 9, 12, 24, 26, 29, 32, 33 IFS has consistently been described as an axial measurement, but this measurement may not capture the actual IFS due to the curvature of the two bones that exists outside of the axial plane (i.e. height differences between the apex of the lesser trochanter and the apex of the ischium). Measurement of the three-dimensional (3D) distance between the lesser trochanter and ischium in asymptomatic controls would provide baseline data and additional volumetric perspective on IFS which may improve our understanding of symptoms consistent with IFI. 
More recent studies have measured reduced IFS during adduction, external rotation, and extension, using MRI, ${ }^{15,28}$ ultrasound, ${ }^{8}$ or direct measurements using calipers or straight rulers on cadavers. ${ }^{20,30}$ Clinicians have also started to incorporate examinations, such as the long-stride walking test, to better diagnose IFI. ${ }^{11}, 13$ However, the specific relationship between dynamic and static measurements of IFS is not yet known. Perhaps most importantly, data are not available to describe how IFS varies as the hip articulates during weight-bearing activities, which are most relevant to understanding the pathomechanics of IFI.

Known variations in pelvic and femoral anatomy between sexes may play a role in decreased IFS and increased prevalence of IFI in females. ${ }^{7}, 24,26,34$ Most of the studies that have measured IFS were composed primarily, or entirely, of female subjects. ${ }^{2,12,26,32,33}$ Here, investigators have assessed radiographic measurements, such as intertuberous distance, ischial angle, inclination angle, and femoral neck version, and have found that these metrics are correlated with IFI. However, the findings across studies have not been consistent. $6,12,24,33$ While these measurements of femoral and pelvic morphology improve our understanding of hip pathology, impingement is a dynamic process that may not be explained by radiographic measurements alone. ${ }^{18}$ Measurements of IFS obtained during dynamic activities, relative to the subject's underlying 3D anatomy, would clarify the role of morphology and kinematics in predisposing females to develop symptomatic IFI more frequently than males.

Dual fluoroscopy (DF) is coupled with CT generated 3D models to quantify in-vivo motion of bones with sub-millimeter and sub-degree accuracy. ${ }^{17}$ This technique displays femur and pelvis motion relative to the subject's 3D anatomy. Using DF of asymptomatic subjects during activities of daily living, the objectives of this study were to: 1) evaluate the relationship of dynamic measurements of IFS and hip kinematics, 2) compare IFS measured from DF and axial MRI between males and females, and 3) investigate the association of morphological measurements obtained from axial MR images and dynamic measurements of IFS. We hypothesized that: 1) IFS would be significantly decreased during dynamic activities when the hip is in adduction, extension, or external rotation as compared to axial measurements from MRI, 2) IFS would be reduced in females when compared to males, and 3) IFS during dynamic activities would be correlated to morphological measurements of the hip.

\section{METHODS}

\section{Subjects}

Eighteen young, asymptomatic, recreationally active adults (five females), recruited via word-of-mouth, provided informed consent in this study approved by the Institutional Review Board. Subjects were pain-free and had no history of surgery to their lower limbs. Measurements of the lateral center edge angle (LCEA) and alpha angle were obtained using an anterior-posterior radiograph (A.E.A.). Using these measurements, subjects were screened for radiographic signs of hip pathoanatomy, such as acetabular dysplasia (LCEA $<25^{\circ}$ ) or femoroacetabular impingement (alpha angle $>55^{\circ}$ for cam-type or LCEA $>39^{\circ}$ for pincer-type). ${ }^{25,} 35$ Normal hip morphology was confirmed using MR images. Screening 
excluded seven subjects, leaving eleven subjects. The side to be imaged was chosen randomly to ensure equal distribution between right and left hips (six right).

\section{Imaging and Data Collection}

CT arthrography images of the hip were acquired for each subject using a 128-section single-source CT machine (SOMATOM Definition, Siemens Healthcare, Munich, Germany). The scan included the entire pelvis and proximal femur ( $1 \mathrm{~mm}$ slices, $120 \mathrm{kVp}$, 200-400 mAs) and the distal femur ( $3 \mathrm{~mm}$ slices, $120 \mathrm{kVp}, 150 \mathrm{mAs}) .{ }^{10,}{ }^{14}, 17$ Threedimensional reconstructions of the pelvis and proximal and distal femur were generated from segmentation of the CT images using the Amira software (v6.0.1, FEI, Hillsboro, OR).

The custom DF system consisted of two pairs of emitters and image intensifiers mounted to four separate bases (Radiological Imaging Services, Hamburg, PA, USA). The emitters and image intensifiers were arranged around an instrumented treadmill (Bertec Corporation, Columbus, $\mathrm{OH}, \mathrm{USA}$ ) to image the hip joint during dynamic activities. Settings for the DF system were adjusted manually to produce images with high contrast and signal-to-noise ratio for each subject. Settings ranged from $78-92 \mathrm{kVp}$ to $1.9-3.2 \mathrm{mAs}$ with a camera exposure of 5-7 ms under continuous fluoroscopy. ${ }^{10,17} \mathrm{DF}$ data was acquired at $100 \mathrm{~Hz}$ and analyzed for five activities (Figure 1), including standing, internal rotation, external rotation, and level and incline $\left(5^{\circ}\right)$ treadmill walking at a self-selected speed $(1.3 \pm 0.1 \mathrm{~m} / \mathrm{s})$. Selfselected speed was determined based on a timed over-ground walk of known distance (139 $\mathrm{m})$. The bone segmentations served as input to model-based markerless tracking, ${ }^{5} \mathrm{a}$ validated method which aligned 3D models and DF images to calculate in-vivo hip motion. 17

T1-weighted, gradient-echo MRI scans were acquired in the coronal plane for each subject using a Siemens Trio 3T MRI Scanner (imaging matrix, $448 \times 448$; in-plane resolution, $0.9375 \times 0.9375 \mathrm{~mm}$; slice thickness, $1 \mathrm{~mm}$; echo time (TE), $2 \mathrm{~ms}$; repetition time (TR), 5 $\mathrm{ms}$; and flip angle, $12^{\circ}$ ). Images of the width of the body were acquired in three or four contiguous sections, as necessary, to image from the $12^{\text {th }}$ thoracic vertebra (T12) to below the articulating surface of the knee. Hook and loop fastener straps were placed around the feet of each subject to ensure that the toes were pointing upward and were approximately hip width apart. Coronal images were reconstructed in the axial plane. The lesser trochanter and distal ischium of the hip imaged with DF were segmented from an axial section of the MRI using Amira. The resulting 3D surfaces were smoothed and decimated to reduce edge effects.

Measurements of pelvic and femoral anatomy were acquired from the MRI images by a board-certified orthopaedic surgeon (T.G.M.). Specifically, femoral and lesser trochanteric version, ${ }^{12}$ ischial angle,${ }^{6}$ and intertuberous distance ${ }^{24}$ were measured from axial images using Horos (v2.0.1, www.horosproject.org). ${ }^{12}$ Inclination angle, ${ }^{33}$ or femoral neck-shaft angle, was measured from coronal mages using IntelliSpace PACS (v4.4, Philips Heathcare, Bothell, WA). Two board-certified orthopaedic surgeons (S.K.A., T.G.M.) measured IFS (Figure 2A) from axial MRI images, these measurements were averaged for all analyses. ${ }^{32}$ 


\section{Data and Statistical Analysis}

Averaged axial measurements of IFS were compared to 3D bone-to-bone distance measurements of IFS based on surface reconstructions from MRI (Figure 2B) and dynamic activities (Figures 2C-2D). For 3D measurements, the region of the proximal femur representing the lesser trochanter was isolated from the 3D surface of the femur using plots of principal curvature. Nodes representing the surface of this isolated region were selected for analysis. Distances between these nodes and the nearest nodes on the ischium were output from PostView. ${ }^{23}$ The minimum nodal distance was identified as the IFS in Matlab (v7.10, The MathWorks, Natick, MA).

The projections of the 3D vector that quantified IFS based on the surfaces generated from MR images were calculated automatically for the axial and coronal planes. These planar projections ignored the superior-inferior and anterior-posterior components of the overall measurement, respectively. The axial and coronal projections of the $3 \mathrm{D}$ vector were compared to the magnitude of the 3D vector (i.e. IFS) as well as the axial MRI slice-based IFS measurement made manually.

For each dynamic activity, motions calculated from DF were applied to 3D CT surface reconstructions in PostView (v 1.9.0, www.febio.org), ${ }^{23}$ and bone-to-bone distance of IFS was measured for each frame of the activity. The minimum, maximum, and range of IFS were determined. Since the internal and external rotations were captured as separate activities, the range of IFS was reported as the difference between the maximum distance observed during internal rotation and the minimum distance observed during external rotation. Similarly, only one minimum and one maximum IFS measurement was reported between the two activities.

Hip joint angles were calculated in Matlab for each dynamic activity using data output from model-based markerless tracking and landmark-based coordinate systems applied to the femur and pelvis. ${ }^{17,} 36$ Joint angles from the standing trial were subtracted from the joint angles for each of the dynamic activities. This was done to control for anatomical differences between subjects and to approximate the kinematic measurements observed clinically using a goniometer in reference to a neutral position, as would be described without further knowledge of femoral rotation or pelvic tilt from CT and markerless tracking. Data from level and incline walking activities were normalized in length from heelstrike to heel-strike to allow for comparisons between subjects walking at different selfselected speeds.

Demographic data, radiographic measurements, and joint angles were evaluated for normality using the Shapiro-Wilk Normality Test. Inter-observer reliabilities were evaluated with the intraclass correlation coefficient (ICC) using a two-way random effects single measurement model. The minimum, maximum, and range of IFS for MRI and DF-based static and dynamic activities were compared using a paired Student's T test. Analysis of sexbased differences was completed using a two sample Student's T test. Correlations were computed using Pearson's product-moment correlation. 95\% confidence intervals (CI) of measurements of IFS and correlation statistics were calculated using the bootstrap resampling procedure. A linear mixed effects model was employed to control for the effects 
of each subject performing multiple activities and the two- and three-way interactions between the three joint angle measurements. The data was mean centered to allow for interpretation of the main effects while accounting for interactions and scaled by the standard deviation to eliminate the effects of the observed range of motion. ${ }^{1,4,21}$ Multiple comparisons were controlled for with the Holm-Bonferroni correction for multiplicity; unless otherwise mentioned, reported p-values have been adjusted. Static and dynamic measurements of IFS were reported as mean $(95 \% \mathrm{CI})$, while demographic data and other population descriptors were represented as mean \pm standard deviation. All statistical analysis was completed in R (www.R-projects.org). ${ }^{31}$

\section{RESULTS}

\section{Demographics and Radiographic Measurements}

The eleven recruited subjects (five female) had a mean age of $23 \pm 2$ years, height of $173.3 \pm 10.4 \mathrm{~mm}$, weight of $63.8 \pm 10.9 \mathrm{~kg}$, and BMI of $21.1 \pm 1.9 \mathrm{~kg} / \mathrm{m}^{2}$. The male and female populations were not significantly different in terms of age or BMI, but the males were taller and heavier than the females (height: $182.0 \pm 2.9$ vs. $162.9 \pm 3.6 \mathrm{~mm}, \mathrm{p}<0.001$, weight: $71.4 \pm 7.2$ vs. $54.7 \pm 6.5 \mathrm{~kg}, \mathrm{p}=0.009)$. Considering all subjects, mean femoral anteversion was $9 \pm 7^{\circ}$, lesser trochanteric retroversion was $19 \pm 12^{\circ}$, inclination angle was $135 \pm 3^{\circ}$, ischial angle was $130 \pm 6^{\circ}$, and intertuberous distance was $133 \pm 13 \mathrm{~mm}$. Intertuberous distance was larger in females than males ( $144 \pm 8$ vs. $124 \pm 8 \mathrm{~mm}, \mathrm{p}=0.011)$, but all other radiographic measurements were not significantly different between males and females. The interobserver reliability of IFS measurements had an ICC of $0.93(0.77-0.98)(\mathrm{p}<0.001)$.

\section{Measurements of IFS - Supine and Standing}

Axial MRI measurements of IFS in a neutral supine position were not significantly different than 3D measurements derived from DF with the subject in a standing position $(\mathrm{p}=0.255)$, but were larger than those from the 3D reconstructions of the supine MRI $(\mathrm{p}=0.005)$ (Table 1). Minimum IFS during standing was significantly smaller in females than males, but differences were not significant when measuring IFS from supine MRI (either from an axial slice or the 3D MRI surface reconstruction) (Table 1).

The axial projection of the 3D measurement of IFS from the supine MRI surface reconstructions was $20.2(17.1-23.4) \mathrm{mm}$, which omitted any superior-inferior distance. The coronal projection was $19.3(16.1-22.7) \mathrm{mm}$, which omitted any anterior-posterior distance. These axial and coronal projections accounted for $94(93-95) \%$ and $89(86-93) \%$ of the 3D measurement, respectively $(\mathrm{p}=0.028)$. Between sexes, the coronal projection of the $3 \mathrm{D}$ measurement was a significantly better representation of the 3D measurement of supine IFS for males than for females ( $94(92-96) \%$ vs. $84(82-86) \%, \mathrm{p}=0.007)$. The axial and coronal projections of the 3D measurement were not significantly different for males. However, for females, the axial projection was a significantly better representation of the 3D measurement of IFS than that of the coronal projection (93 (92-95)\% vs. $84(82-86) \%, \mathrm{p}=0.007)$. 


\section{Measurements of IFS - Internal and External Rotation}

Minimum IFS during external rotation was significantly smaller than axial supine MRI measurements $(\mathrm{p}<0.001)$ (Table 2). Minimum IFS occurred during external rotation, while maximum IFS occurred during internal rotation. Compared to axial supine MRI measurements, both males and females had significantly smaller IFS during external rotation (males, $\mathrm{p}=0.008$; females, $\mathrm{p}=0.011$ ). However, the maximum, minimum, and range of IFS during internal and external rotation were not significantly different between males and females (Table 2).

\section{Measurement of IFS - Level Walking}

Minimum IFS during level walking was significantly smaller than axial supine MRI measurements ( $\mathrm{p}=0.007$ ) (Table 3). Minimum IFS occurred during terminal stance before hip flexion increased with the forward swing of the leg (45 (42-48)\% gait), while maximum IFS occurred during mid-swing when hip flexion was increased in preparation for heel strike (84 (83-86)\% gait). Compared to axial supine MRI measurements, females had significantly smaller IFS during level walking $(\mathrm{p}=0.011)$. When compared to males, females had a significantly smaller minimum IFS and significantly larger range of IFS (Table 3).

\section{Measurement of IFS - Incline Walking}

Minimum IFS during incline walking was significantly smaller than axial supine MRI measurements ( $\mathrm{p}=0.004$ ) (Table 4). Minimum IFS occurred during terminal stance (49 (46$51) \%$ gait), while maximum IFS occurred during mid-swing (88 (86-90)\% gait). Compared to axial supine MRI measurements, females had significantly smaller IFS during incline walking ( $\mathrm{p}=0.008$ ). Minimum IFS was significantly smaller in females than males (Table 4).

\section{Comparisons of IFS - Activities and Joint Angles}

For the dynamic activities observed, minimum IFS occurred during the external rotation pivot for ten of eleven subjects and incline walking for the remaining subject, who was a female. The minimum IFS observed during the external rotation activity was significantly smaller than level walking $(\mathrm{p}=0.007)$ or incline walking $(\mathrm{p}=0.006)$. The maximum IFS observed during the dynamic activities was significantly greater for incline walking than level walking ( $\mathrm{p}=0.001$; see Tables $3-4$ for IFS values). The range of IFS was significantly smaller during level walking when compared to either rotation $(\mathrm{p}=0.033)$ or incline walking $(\mathrm{p}=0.045)$ (see Tables $2-4$ for IFS values). Beyond the reported IFS measurements, visualization of femur-pelvis motion during these activities demonstrated the truly dynamic nature of IFS (Supplemental Video 1).

Joint angles for males and females were not significantly different in any direction at minimum or maximum IFS during any activity, including the standing trial (unadjusted $\mathrm{p}>0.027$ ). Compared to the joint angles at maximum IFS, minimum IFS presented at significantly greater adduction and extension angles for all activities and greater external rotation for rotation and incline walking activities (Table 5). Both minimum and maximum IFS occurred earlier in the gait cycle for level walking than for incline walking (minimum IFS, $\mathrm{p}=0.021$; maximum IFS, $\mathrm{p}=0.011$ ). 


\section{Correlations}

The minimum IFS observed for each subject (considering all activities and trials) was moderately to strongly correlated with axial IFS from supine MRI (95\% CI, 0.33-0.97) (Figure 3). However, other MRI-based measurements of anatomy (femoral anteversion, lesser trochanteric retroversion, inclination angle, ischial angle, and intertuberous distance) were not significantly correlated to either MRI based measurement of IFS or to the overall minimum observed dynamic IFS ( $r<0.53$, unadjusted $\mathrm{p}>0.090$ ).

For all observed activities, the range of motion was $16 \pm 6^{\circ}$ for abduction-adduction, $62 \pm 8^{\circ}$ for internal-external rotation, and $53 \pm 4^{\circ}$ flexion-extension. Considering the joint angle measurements at minimum and maximum IFS for all dynamic activities, dynamic IFS was significantly affected by abduction-adduction $(\beta=0.22(0.16-0.29))$, internal-external rotation $(\beta=0.66(0.54-0.75))$, and flexion-extension $(\beta=0.21(0.12-0.29))(p<0.001$ for all $)$. The two-way interaction between internal-external rotation and flexion-extension was also significant $(\beta=0.21(0.11-0.33), \mathrm{p}<0.001)$, the effects of all other joint angle interactions were not significant $(\beta \unlhd 0.03, \mathrm{p}>0.212)$.

\section{DISCUSSION}

While IFI is a dynamic process, many clinical diagnoses rely on measurements from static imaging acquired in a supine, neutral position, which may not accurately represent IFS during dynamic activities. Using DF of asymptomatic subjects during activities of daily living, the objectives of this study were to: 1) evaluate the relationship of dynamic measurements of IFS and hip kinematics, 2) compare IFS measured from DF and axial MRI between males and females, and 3) investigate the association of morphological measurements obtained from axial MR images and dynamic measurements of IFS. We confirmed that relative IFS decreased with increased adduction, external rotation, and extension angles of the hip. We also confirmed that minimum IFS was decreased during dynamic activities, as compared to static measurements from 3D reconstructions or an axial slice from MRI. Further, we found that IFS was narrower in females when compared to males, but only during standing and level and incline walking. Finally, only one measurement of pelvic morphology showed significant differences between sexes, indicating that the sex-based differences in dynamic IFS may be due to a combination of subtle variations in both kinematics and anatomy. Based on the strength of the relationship between IFS and hip kinematics, our results suggest that IFS is reduced most during extension. However, variations in kinematics or morphology did not elucidate the sex-based differences in dynamic IFS. Collectively, our results provide insight into how IFS is affected by motion in asymptomatic subjects, thus providing important baseline data and additional volumetric perspective on IFS that could be used to improve our understanding of symptoms consistent with IFI.

IFS, as measured axially on supine MRI in our study, had good inter-rater reliability and corresponded well with ICC values reported in the literature. ${ }^{33}$ Additionally, our measures of IFS were similar to those previously reported. For example, studies with a relatively equal distribution of males and females (40-70\% female) reported IFS between 23 and $28 \mathrm{~mm}$. $9,12,24$ In addition, studies with predominantly (75-85\%) female populations measured IFS 
to be slightly lower at $21-22 \mathrm{~mm},{ }^{19}, 26,33$ which corresponds to our measurements of IFS in females. While several studies have observed smaller IFS in females, only one has found this difference to be significant. ${ }^{24}$ The lack of significant differences in the literature could be due to the relatively small sample sizes used in evaluation of IFS or possibly due to limited sex-based differences that can be visualized on axial slices of MRI images.

Minimum IFS measured dynamically was smaller than that from MRI-based measurements with the subject supine. Previous studies have evaluated range of IFS during dynamic passive motion, including up to $30^{\circ}$ abduction-adduction 8,30 and $100^{\circ}$ internal-external rotation $8,20,28,30$ at either neutral or slight extension, as measured using a goniometer or protractor. Ranges of abduction-adduction and internal-external rotation that incurred as a result of the dynamic activities investigated in our study were smaller than has been previously evaluated. However, our range of flexion-extension was larger. Despite differences in hip kinematics, our measurements of minimum, maximum, and range of IFS were similar to prior studies which have observed overall ranges of IFS between 19 and 29 $\mathrm{mm},{ }^{8,20,28}$ and reductions from neutral IFS of 5 and $14 \mathrm{~mm} .{ }^{20,30}$ In particular, we observed the average overall range of dynamic IFS to be $33 \mathrm{~mm}$ with reductions from axial IFS of 15 $\mathrm{mm}$.

Correlations between IFS and hip kinematics measured in our study suggest that clinical exams and gait tests should emphasize positions that place the hip into greater extension, rather than isolated adduction or internal-external rotation, as has been observed previously. 20, 28 While axial measurements of IFS were moderately to strongly correlated with the minimum observed dynamic IFS, the $95 \%$ CI ranged from a negligible to a very strong relationship, indicating that use of axial IFS to estimate dynamic IFS may be inconsistent and unreliable (Figure 3). For these reasons, we advocate for the use of clinical exams, such as the long stride walking test, described by Gómez-Hoyos and colleagues, in addition to imaging in the diagnosis of IFI. ${ }^{11}$ Nevertheless, it is important to note that our recommendation for the long stride walking test is only based on analysis of asymptomatic subjects, not symptomatic IFI patients.

Despite there being no significant differences in kinematics or axial IFS between sexes, differences in minimum IFS during standing and both walking activities were significant. This finding, and previous results, suggests that in addition to the kinematic position of the joint, the anatomical appearance of the femur and pelvis also affects dynamic IFS. 6, 12, 33 The sexual dimorphism associated with IFI may be partially explained by increased intertuberous distance in females. ${ }^{6,24}$ While this anatomical measurement may not have a significant effect on IFS in the supine position, the increased pelvic width may cause more substantial decreases in IFS dynamically. However, for our population, we did not observe significant correlations between any of the observed radiographic measurements and IFS, either statically or dynamically. Reduced IFS with dynamic motion, especially in daily activities, such as walking, demonstrates the repetitive nature of impingement that may lead to tissue damage and pain in patients with IFI. The specific reductions of IFS in females during these dynamic activities may further help to explain the predominance of IFI in females identified in the literature. ${ }^{3,11-13,27,32}$ 
When compared to 3D magnitude measurements of IFS, slice-based measurements likely overestimate the space between the lesser trochanter and ischium, while projection-based measurements likely underestimate this space. These measurement biases are a result of the inability to capture the spatial relationship of structures misaligned with the $2 \mathrm{D}$ imaging plane (e.g., consider an axial measurement of IFS given the superior-inferior offset between the lesser trochanter and ischium in Figure 2B). Our results from DF, which are based on the 3D relationship between the curvature of the lesser trochanter and the ischium and not on projection- or slice-based measurements, suggest that coronal-based measurements of IFS may be relevant to our understanding of IFS. Park et al. concluded that anterior-posterior radiographs (i.e. a coronal projection) could be used as an alternative method to measure IFS. ${ }^{26}$ However, we observed a larger anterior-posterior distance between the lesser trochanter and ischium in females. This difference would likely not have been evident with the use of projection based imaging or when considering a high proportion of females (26:4 female-to-male ratio in the study by Park et al.). The larger anterior-posterior distance observed in females in our study would likely result in greater reductions in IFS during dynamic motions, especially extension. Further investigation is necessary to determine if an increase in the anterior-posterior offset between the lesser trochanter and ischium can help to explain the predominance of symptomatic IFI in females.

\section{Limitations}

Measurements of bone-to-bone distance from DF provide accurate data to study IFS under dynamic, weight-bearing activities. However DF and associated data processing are timeand labor-intensive, which limited the number of participants that could be analyzed. Nevertheless, our sample size of 11 hips is similar to previous studies evaluating IFS at varied positions, where sample sizes ranged from 10 to 16 subjects. ${ }^{8,20,30} \mathrm{We}$ acknowledge that our statistical comparisons between sexes are likely inadequately powered, and thus, we advocate for caution when generalizing the results of our study.

Our control subjects were screened for abnormalities by a single anterior-posterior radiograph. It is possible that they had morphometric deformities to the femur and/or pelvis that were not observed on the anterior-posterior film. Measurements of both femoral neck version and inclination angles are increased in hips with symptomatic IFI. ${ }^{6,}{ }^{12}, 33 \mathrm{In}$ comparison to controls in previous studies, we observed decreased femoral neck version and increased inclination angles. $6,12,33$ While these measurements were within a range considered to be normal, ${ }^{12,22,33}$ the dissimilarity in femoral neck version and similarity in inclination angle may convolute the interpretation of our IFS measurements in terms of patients with IFI. Importantly, many studies reporting IFS or other radiographic measurements associated with IFI only retrospectively evaluated images on the basis of any reported hip pain and existence of muscle edema. ${ }^{2}$, 6, 12, 26, 32, 33 Many of the control subjects in these prior studies had contralateral hip pain which may have affected general morphology of the hip or masked ipsilateral hip pain. In contrast, our controls were recruited prospectively; those with a history of hip pain were excluded, which we believe is a major strength of our study compared to prior research. 
Our measurement of IFS in asymptomatic individuals during dynamic activities may not accurately represent the variability in IFS in symptomatic patients with IFI due to altered morphology or motion patterns. In addition, symptomatic patients may have different demographics (e.g. sex, age) compared to the young, athletic cohort studied herein. However, there exists mixed conclusions in the literature regarding morphological alterations associated with IFI (i.e., morphological variations may be subtle or are not defined with current measurements of hip morphology). ${ }^{6,12,24,30,33}$ Further, there has been no indication to date that IFI patients move with altered motion patterns. Our results provide baseline data with which to compare to symptomatic patients analyzed in the future. Importantly, DF displays the motion relative to the subject's 3D femoral and pelvic anatomy reconstructed from a CT scan, which enables investigation of the relationship between hip morphology and motion.

Finally, owed to the use of DF, which does not image soft-tissue, we did not measure dynamic changes in the quadratus femoris space. The quadratus femoris space may be more relevant to the study of symptomatic IFI patients than IFS. Still, a previous cadaver study by Sussman et al. found IFS and quadratus femoris space to reduce similarly from neutral to maximal approximation. ${ }^{30}$ Thus, it may be possible to predict quadratus femoris space from the IFS. Nevertheless, the purpose of this study was to measure IFS dynamically in asymptomatic individuals without hip pathoanatomy so as to provide critical data to understand the limitations of static measurements of this metric, and a framework in which to investigate symptomatic patients in the future.

\section{CONCLUSION}

In this cohort of asymptomatic subjects, dynamic IFS was reduced when compared to supine measurements for walking and rotation activities. Further, there were significant differences in IFS between sexes during walking activities. However neither kinematics nor morphological measurements explain these variations. A subtle combination of kinematic and morphological variations may account for the dynamic variation in IFS. The relationship between IFS and flexion-extension suggests that clinical exams and imaging should emphasize positions that place the hip into greater extension, rather than isolated adduction or external rotation to achieve realization of minimum IFS, as has been recommended previously. ${ }^{28}$ Collectively, these results suggest that measurements of IFS from static imaging, especially in a neutral orientation, are not consistent with dynamic measurements and may not accurately represent decreased IFS during dynamic motion. However, we base this recommendation on analysis of asymptomatic subjects, not symptomatic IFI patients, and thus, additional research is necessary. Future research should include dynamic analyses of hips with symptomatic IFI to understand the dynamic nature of this condition and directly investigate the optimal position to observe minimum IFS. In addition, recognizing the timeintensive nature of dynamic imaging, future research should investigate methods to develop predictive models of IFI that do not require dual fluoroscopy.

\section{Supplementary Material}

Refer to Web version on PubMed Central for supplementary material. 


\section{Acknowledgements}

The authors acknowledge financial support from the National Institutes of Health (R21AR063844, and S10RR026565) and the LS-Peery Discovery Program in Musculoskeletal Restoration. This investigation was supported by the University of Utah PHR and Population Health Research Foundation, with funding in part from the National Center for Research Resources and the National Center for Advancing Translational Sciences, National Institutes of Health, through Grant 5UL1TR001067-02 (formerly 8UL1TR000105 and UL1RR025764). The research content herein is solely the responsibility of the authors and does not necessarily represent the official views of the National Institutes of Health or LS-Peery Program.

\section{REFERENCES}

1. Aiken LS, West SG, Reno RR. Multiple regression: Testing and interpreting interactions: Sage; 1991.

2. Ali AM, Teh J, Whitwell D, Ostlere S. Ischiofemoral impingement: a retrospective analysis of cases in a specialist orthopaedic centre over a four-year period. Hip Int. 2013;23(3):263-268. [PubMed: 23760746]

3. Ali AM, Whitwell D, Ostlere SJ. Case report: imaging and surgical treatment of a snapping hip due to ischiofemoral impingement. Skeletal Radiol. 2011;40(5):653-656. [PubMed: 21207021]

4. Bates D, Mächler M, Bolker B, Walker S. Fitting Linear Mixed-Effects Models Using lme4. Journal of Statistical Software. 2015;67(1):1-48.

5. Bey MJ, Zauel R, Brock SK, Tashman S. Validation of a new model-based tracking technique for measuring three-dimensional, in vivo glenohumeral joint kinematics. J Biomech Eng. 2006;128(4): 604-609. [PubMed: 16813452]

6. Bredella MA, Azevedo DC, Oliveira AL, et al. Pelvic morphology in ischiofemoral impingement. Skeletal Radiol. 2015;44(2):249-253. [PubMed: 25371087]

7. Brinckmann P, Hoefert H, Jongen HT. Sex differences in the skeletal geometry of the human pelvis and hip joint. J Biomech. 1981;14(6):427-430. [PubMed: 7263735]

8. Finnoff JT, Bond JR, Collins MS, et al. Variability of the ischiofemoral space relative to femur position: An ultrasound study. Pm r. 2015;7(9):930-937. [PubMed: 25772723]

9. Finnoff JT, Johnson AC, Hollman JH. Can ultrasound accurately assess ischiofemoral space dimensions? A validation study. Pm r. 2016; [Epub ahead of print].

10. Fiorentino NM, Kutschke MJ, Atkins PR, Foreman KB, Kapron AL, Anderson AE. Accuracy of functional and predictive methods to calculate the hip joint center in young non-pathologic asymptomatic adults with dual fluoroscopy as a reference standard. Ann Biomed Eng. 2016;44(7): 2168-2180. [PubMed: 26645080]

11. Gómez-Hoyos J, Martin RL, Schröder R, Palmer IJ, Martin HD. Accuracy of 2 clinical tests for ischiofemoral impingement in patients with posterior hip pain and endoscopically confirmed diagnosis. Arthroscopy. 2016;32(7):1279-1284. [PubMed: 27020393]

12. Gómez-Hoyos J, Schroder R, Reddy M, Palmer IJ, Martin HD. Femoral neck anteversion and lesser trochanteric retroversion in patients with ischiofemoral impingement: A case-control magnetic resonance imaging study. Arthroscopy. 2016;32(1):13-18. [PubMed: 26358634]

13. Hatem MA, Palmer IJ, Martin HD. Diagnosis and 2-year outcomes of endoscopic treatment for ischiofemoral impingement. Arthroscopy. 2015;31(2):239-246. [PubMed: 25278353]

14. Henak CR, Kapron AL, Anderson AE, Ellis BJ, Maas SA, Weiss JA. Specimen-specific predictions of contact stress under physiological loading in the human hip: validation and sensitivity studies. Biomech Model Mechanobiol. 2014;13(2):387-400. [PubMed: 23736783]

15. Johnson AC, Hollman JH, Howe BM, Finnoff JT. Variability of ischiofemoral space dimensions with changes in hip flexion: an MRI study. Skeletal Radiol. 2017;46(4):59-64. [PubMed: 27738747]

16. Johnson KA. Impingement of the lesser trochanter on the ischial ramus after total hip arthroplasty. Report of three cases. J Bone Joint Surg Am. 1977;59(2):268-269. [PubMed: 845219]

17. Kapron AL, Aoki SK, Peters CL, et al. Accuracy and feasibility of dual fluoroscopy and modelbased tracking to quantify in vivo hip kinematics during clinical exams. J Appl Biomech. 2014;30(3):461-470. [PubMed: 24584728] 
18. Kassarjian A Signal abnormalities in the quadratus femoris muscle: Tear or impingement? Am J Roentgenol. 2008;190(6):W379-W379. [PubMed: 18492883]

19. Khodair SA, Ghieda UE, Elsayed AS. Ischiofemoral impingement syndrome: Spectrum of MRI findings in comparison to normal subjects. Egypt J Radiol Nucl Med. 2014;45(3):819-824.

20. Kivlan BR, Martin RL, Martin HD. Ischiofemoral impingement: defining the lesser trochanterischial space. Knee Surg Sports Traumatol Arthrosc. 2017;25(1):72-76. [PubMed: 26869034]

21. lmerTest: Tests in Linear Mixed Effects Models [computer program]. Version; 2015.

22. Lequesne M, Morvan G. Description of the potential of an arthrometer for standard and reduced radiographs suitable to measurement of angles and segments of hip, knee, foot and joint space widths. Joint Bone Spine. 2002;69(3):282-292. [PubMed: 12102275]

23. Maas SA, Ellis BJ, Ateshian GA, Weiss JA. FEBio: finite elements for biomechanics. J Biomech Eng. 2012;134(1):011005. [PubMed: 22482660]

24. Maras Ozdemir Z, Aydingoz U, Gormeli CA, Sagir Kahraman A. Ischiofemoral space on MRI in an asymptomatic population: Normative width measurements and soft tissue signal variations. Eur Radiol. 2015;25(8):2246-2253. [PubMed: 25680726]

25. Notzli HP, Wyss TF, Stoecklin CH, Schmid MR, Treiber K, Hodler J. The contour of the femoral head-neck junction as a predictor for the risk of anterior impingement. J Bone Joint Surg Br. 2002;84(4):556-560. [PubMed: 12043778]

26. Park S, Lee HY, Cuong PM, et al. Supine versus standing radiographs for detecting ischiofemoral impingement: A propensity score-matched analysis. Am J Roentgenol. 2016;206(6):1253-1263. [PubMed: 27070951]

27. Patti JW, Ouellette H, Bredella MA, Torriani M. Impingement of lesser trochanter on ischium as a potential cause for hip pain. Skeletal Radiol. 2008;37(10):939-941. [PubMed: 18682931]

28. Singer A, Clifford P, Tresley J, Jose J, Subhawong T. Ischiofemoral impingement and the utility of full-range-of-motion magnetic resonance imaging in its detection. Am J Orthop. 2014;43(12):548551. [PubMed: 25490008]

29. Singer AD, Subhawong TK, Jose J, Tresley J, Clifford PD. Ischiofemoral impingement syndrome: a meta-analysis. Skeletal Radiol. 2015;44(6):831-837. [PubMed: 25672947]

30. Sussman WI, Han E, Schuenke MD. Quantitative assessment of the ischiofemoral space and evidence of degenerative changes in the quadratus femoris muscle. Surg Radiol Anat. 2013;35(4): 273-281. [PubMed: 23076730]

31. R: A Language and Environment for Statistical Computing [computer program]. Version 3.1.2. Vienna, Austria: R Foundation for Statistical Computing; 2014.

32. Torriani M, Souto SCL, Thomas BJ, Ouellette H, Bredella MA. Ischiofemoral impingement syndrome: An entity with hip pain and abnormalities of the quadratus femoris muscle. Am J Roentgenol. 2009;193(1):186-190. [PubMed: 19542413]

33. Tosun O, Algin O, Yalcin N, Cay N, Ocakoglu G, Karaoglanoglu M. Ischiofemoral impingement: evaluation with new MRI parameters and assessment of their reliability. Skeletal Radiol. 2012;41(5):575-587. [PubMed: 21874607]

34. Unnanuntana A, Toogood P, Hart D, Cooperman D, Grant RE. Evaluation of proximal femoral geometry using digital photographs. J Orthop Res. 2010;28(11):1399-1404. [PubMed: 20872573]

35. Wiberg G Studies on Dysplastic Acetabula and Congenital Subluxation of the Hip Joint: With Special Reference to the Complication of Osteo-arthritis: Karolinska Institutet, Orthopedic Clinic; 1939.

36. Wu G, Siegler S, Allard P, et al. ISB recommendation on definitions of joint coordinate system of various joints for the reporting of human joint motion-part I: ankle, hip, and spine. J Biomech. 2002;35(4):543-548. [PubMed: 11934426] 


\section{What is known about the subject}

Ischiofemoral impingement (IFI) is a cause of hip pain due to compression of soft tissue structures between the lesser trochanter and ischium that has predominately been diagnosed in females. Prior studies have identified the ischiofemoral space (IFS) as the distance between the lesser trochanter and ischium as measured, most commonly, on supine axial magnetic resonance imaging (MRI) slices. 2, 6, 9, 12, 24, 26, 29, 32, 33 Results from these prior studies suggest that IFS is reduced in females. 2, 6, 32, 33 However, only one prior study has found statistically significant differences in IFS measurements between sexes. ${ }^{24}$ Hips with increased femoral neck version, ${ }^{6,12}$ increased angle between femoral neck version and lesser trochanter version, ${ }^{12}$ increased intertuberous distance ${ }^{24}$, or increased inclination angle ${ }^{33}$ are thought to have reduced IFS or be at greater risk for IFI. However, there is no consensus regarding which morphometrics should be of most interest in the context of IFI. More recent research has measured IFS at specific rotations of the femur, and showed a decrease in IFS with adduction, external rotation, and extension. ${ }^{8,20,28,30}$ However, these studies did not quantify IFS during dynamic activities, which are most relevant to understanding the pathomechanics of IFI. 


\section{What this study adds to existing knowledge}

We established baseline measurements of IFS in asymptomatic controls during activities of daily living by coupling three-dimensional (3D) computed tomography (CT) models with dual fluoroscopy (DF) images to visualize in-vivo hip motion. We then compared dynamic measurements of IFS to those obtained from supine MRI, based on axial images and 3D models. We found that 2D and 3D measurements from MRI were not significantly different from one another. The planar projections of the 3D MRI measurement were a good approximation for the overall (i.e. Euclidean) distance. However the larger anterior-posterior distance in females resulted in reduced accuracy for the coronal-based measurement. Differences between sexes were also observed during dynamic activities. Yet differences were not significant during internal-external rotation or when measuring IFS from supine MRI, either from an axial slice or the 3D surface reconstructions.

Interestingly, neither morphological nor kinematic differences between sexes fully explained differences in the IFS. A stronger relationship between IFS and flexionextension suggest that clinical exams and imaging should place the hip into greater extension, rather than adduction or external rotation as has previously been measured. $8,20,28,30$ Our results indicate that IFS measured from static images, especially in a neutral orientation, may not accurately represent IFS during dynamic motion. 


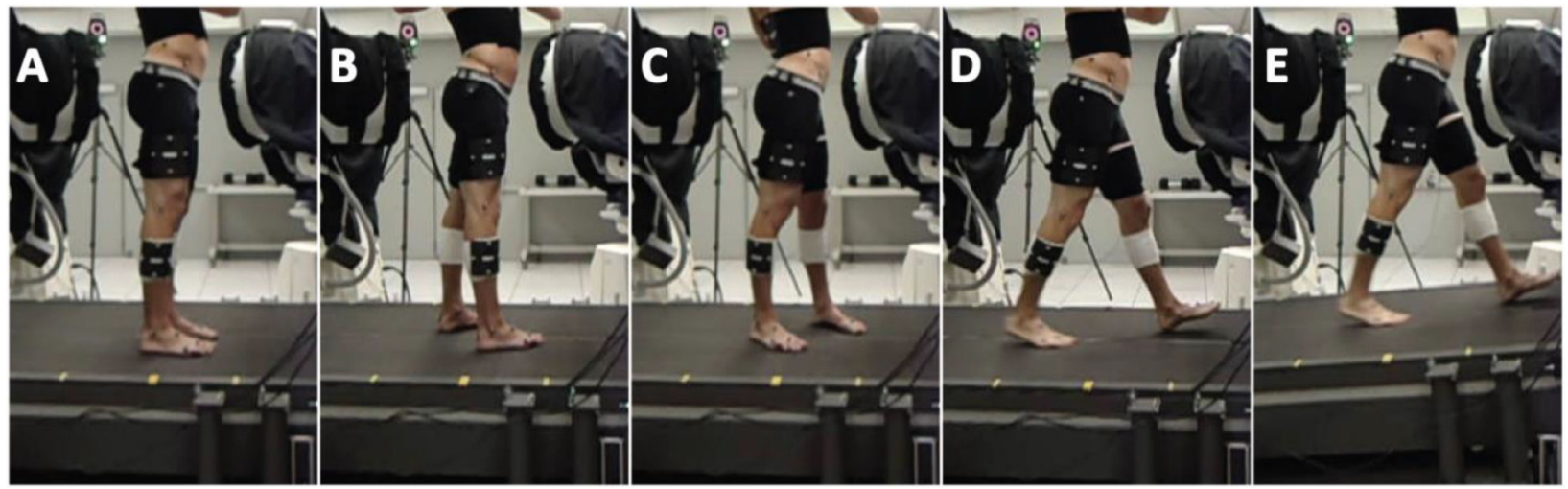

Figure 1:

Representative male subject during dynamic dual fluoroscopy capture of right hip during activities: (A) standing, (B) internal rotation (terminal position shown), (C) external rotation (terminal position shown), (D) level treadmill walk, and (E) incline treadmill walk. Image intensifiers were located anterior and posterior to the subject in the foreground of each image, beam emitters were not visible. 



Figure 2:

Measurement of IFS in a representative female subject using MRI and DF methods. IFS was measured from a single, axial MRI slice (white line) (A). The MRI was then segmented to generate 3D reconstructions of the lesser trochanter and ischium, and IFS was calculated based on the distance between the two surfaces (B). Model-based markerless tracking was used to quantify in-vivo hip position by aligning 3D CT reconstructions with DF images. IFS was calculated based on the distance between the femur and pelvis surfaces for a standing trial and four dynamic activities. Minimum and maximum IFS are shown for the level walking trial (C-D, respectively). 


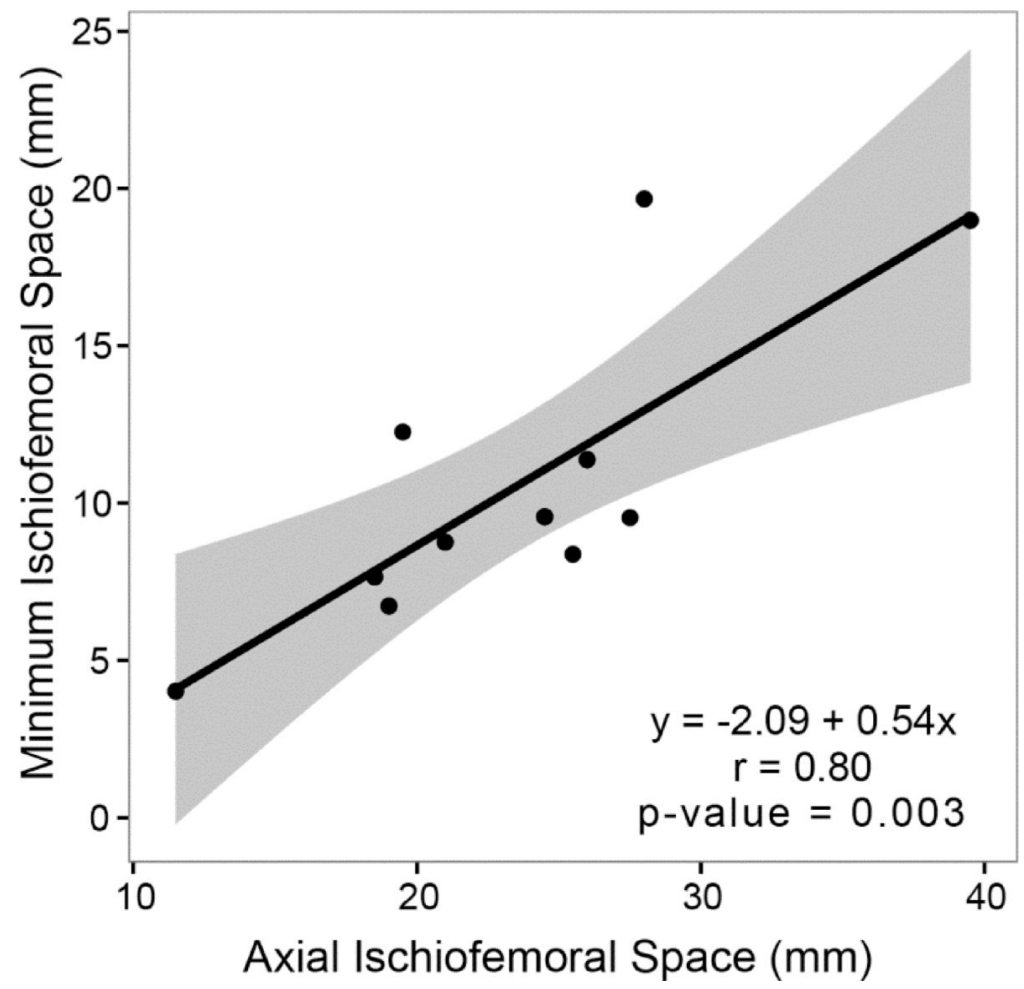

Figure 3:

Correlation plot of minimum measured ischiofemoral space (IFS) for all dynamic activities from dual fluoroscopy (DF) with axial supine magnetic resonance imaging (MRI) IFS. The best fit linear model is represented as the black line and the $95 \%$ confidence interval shown in gray; the correlation statistic and p-value are provided. 


\section{TABLE 1}

IFS Measured on MRI Axial Slices, 3D MRI Surface Reconstructions, and CT Surface Reconstructions from Dual Fluoroscopy $^{a}$

\begin{tabular}{ccccc}
\hline & Cohort & Male & Female & P Value \\
\hline Axial MRI (Supine) & $23.7(19.9-27.9)$ & $26.0(22.1-30.3)$ & $20.9(17.5-24.0)$ & 0.311 \\
3D MRI (Supine) & $21.4(18.3-24.8)$ & $23.8(20.8-26.9)$ & $18.7(15.9-21.3)$ & 0.311 \\
Standing & $26.1(22.3-30.2)$ & $30.4(27.2-33.8)$ & $20.9(19.3-22.3)$ & $0.034^{b}$ \\
\hline
\end{tabular}

${ }^{a}$ Data reported as mean (95\% confidence interval) in mm. IFS, ischiofemoral space; MRI, magnetic resonance imaging; CT, computed tomography.

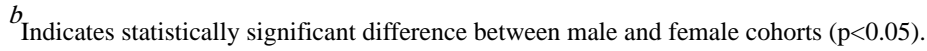


TABLE 2

Ischiofemoral Space during Internal and External Rotation ${ }^{a}$

\begin{tabular}{ccccc}
\hline & Cohort & Male & Female & P Value \\
\hline Axial MRI (Supine) & $23.7(19.9-27.9)$ & $26.0(22.1-30.3)$ & $20.9(17.5-24.0)$ & 0.311 \\
Minimum & $10.8(8.3-13.7)$ & $13.3(10.6-16.0)$ & $7.9(6.6-8.9)$ & 0.145 \\
Maximum & $43.8(39.7-47.9)$ & $45.5(40.9-50.0)$ & $41.8(38.5-45.0)$ & 0.823 \\
Range & $33.0(30.3-35.6)$ & $32.3(30.1-34.2)$ & $33.9(30.7-36.9)$ & 0.823 \\
\hline$a$ \\
\multicolumn{6}{c}{ Data reported as mean (95\% confidence interval) in mm. } \\
$b$ Indicates statistically significant difference between male and female cohorts $(\mathrm{p}<0.05)$.
\end{tabular}




\section{TABLE 3}

Ischiofemoral Space during Level Walking $^{a}$

\begin{tabular}{ccccc}
\hline & Cohort & Male & Female & P Value \\
\hline Axial MRI (Supine) & $23.7(19.9-27.9)$ & $26.0(22.1-30.3)$ & $20.9(17.5-24.0)$ & 0.311 \\
Minimum & $15.5(11.4-19.7)$ & $21.1(18.7-23.6)$ & $8.8(7.5-9.9)$ & $0.001^{b}$ \\
Maximum & $42.9(39.5-46.5)$ & $45.7(42.4-29.1)$ & $39.5(37.4-41.7)$ & 0.087 \\
Range & $27.4(24.7-30.1)$ & $24.7(22.5-26.9)$ & $30.7(28.9-32.4)$ & $0.049^{b}$ \\
\hline
\end{tabular}

${ }^{a}$ Data reported as mean (95\% confidence interval) in $\mathrm{mm}$.

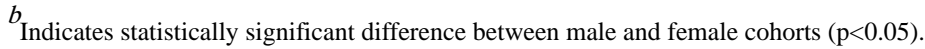




\section{TABLE 4}

Ischiofemoral Space during Incline Walking ${ }^{a}$

\begin{tabular}{ccccc}
\hline & Cohort & Male & Female & P Value \\
\hline Axial MRI (Supine) & $23.7(19.9-27.9)$ & $26.0(22.1-30.3)$ & $20.9(17.5-24.0)$ & 0.311 \\
Minimum & $15.8(11.6-20.1)$ & $21.3(18.9-24.1)$ & $9.1(7.4-10.8)$ & $0.003 b$ \\
Maximum & $48.3(45.1-51.6)$ & $51.3(48.3-54.2)$ & $44.7(42.5-47.0)$ & 0.101 \\
Range & $32.5(29.5-35.2)$ & $30.0(27.1-32.8)$ & $35.6(33.8-37.2)$ & 0.101 \\
\hline
\end{tabular}

${ }^{a}$ Data reported as mean (95\% confidence interval) in $\mathrm{mm}$.

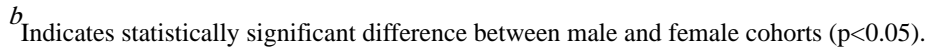


TABLE 5

Comparison of Joint Angles for Minimum and Maximum IFS during Dynamic Activities ${ }^{a}$

\begin{tabular}{cccc}
\hline & Adduction/ Abduction & External/ Internal Rotation & Extension/ Flexion \\
\hline Rotation & $0.006^{b}$ & $<0.001^{b}$ & $<0.0011^{b}$ \\
Level Walking & $<0.001^{b}$ & 0.792 & $<0.001^{b}$ \\
Incline Walking & $0.041^{b}$ & $0.041^{b}$ & $<0.001^{b}$ \\
\hline$a$ & & \\
${ }^{2}$ Data reported as p-value & & \\
$b_{\text {Indicates statistically significant difference between kinematics at minimum and maximum IFS (p<0.05). }}$
\end{tabular}

